\title{
Spiking Neural P Systems with Neuron Division and Budding
}

\author{
Linqiang Pan ${ }^{1,2}$, Gheorghe Păun²,3, Mario J. Pérez-Jiménez ${ }^{2}$ \\ 1 Department of Control Science and Engineering \\ Huazhong University of Science and Technology \\ Wuhan 430074, Hubei, China \\ lqpan@mail.hust.edu.cn, lqpan@us.es \\ 2 Department of Computer Science and Artificial Intelligence \\ University of Sevilla \\ Avda. Reina Mercedes s/n, 41012 Sevilla, Spain \\ marper@us.es \\ 3 Institute of Mathematics of the Romanian Academy \\ PO Box 1-764, 014700 Bucureşti, Romania \\ george.paun@imar.ro, gpaun@us.es
}

Summary. In order to enhance the efficiency of spiking neural $\mathrm{P}$ systems, we introduce the features of neuron division and neuron budding, which are processes inspired by neural stem cell division. As expected (as it is the case for $\mathrm{P}$ systems with active membranes), in this way we get the possibility to solve computationally hard problems in polynomial time. We illustrate this possibility with SAT problem.

\section{Introduction}

Spiking neural $\mathrm{P}$ systems (in short, $\mathrm{SN} \mathrm{P}$ systems) were introduced in [6] in the framework of membrane computing [13] as a new class of computing devices which are inspired by the neurophysiological behavior of neurons sending electrical impulses (spikes) along axons to other neurons. Since then, many computational properties of SN P systems have been studied; for example, it has been proved that they are Turing-complete when considered as number computing devices [6], when used as language generators [3, 1] and also when computing functions [12].

Investigations related to the possibility to solve computationally hard problems by using SN P systems were first proposed in [2]. The idea was to encode the instances of decision problems in a number of spikes which are placed in an arbitrarily large pre-computed system at the beginning of the computation. It was shown that the resulting SN P systems are able to solve the NP-complete problem SAT (the satisfiability of propositional formulas expressed in conjunctive normal form) in a constant time. Slightly different solutions to SAT and 3-SAT by using SN 
P systems with pre-computed resources were considered in [7]; here the encoding of an instance of the given problem is introduced into the pre-computed resources in a polynomial number of steps, while the truth values are assigned to the Boolean variables of the formula and the satisfiability of the clauses is checked. The answer associated with the instance of the problem is thus computed in a polynomial time. Finally, very simple semi-uniform and uniform solutions to the numerical NP-complete problem Subset Sum - by using SN P systems with exponential size pre-computed resources - have been presented in [8]. All the systems constructed above work in a deterministic way.

A different idea of constructing SN P systems for solving NP-complete problems was given in $[10,11]$, where the Subset Sum and SAT problems were considered. In these papers, the solutions are obtained in a semi-uniform or uniform way by using non-deterministic devices but without pre-computed resources. However, several ingredients are also added to SN P systems such as extended rules, the possibility to have a choice between spiking rules and forgetting rules, etc. An alternative to the constructions of $[10,11]$ was given in [9], where only standard SN P systems without delaying rules and having a uniform construction are used. However, it should be noted that the systems described in [9] either have an exponential size, or their computations last an exponential number of steps. Indeed, it has been proved in [11] that a deterministic SN P system of a polynomial size cannot solve an NP-complete problem in a polynomial time unless $\mathbf{P}=\mathbf{N P}$. Hence, under the assumption that $\mathbf{P} \neq \mathbf{N P}$, efficient solutions to $\mathbf{N P}$-complete problems cannot be obtained without introducing features which enhance the efficiency of the system.

In this paper, neuron division and budding are introduced into the framework of SN P systems in order to enhance the efficiency of these systems. We exemplify this possibility with a uniform solution to SAT problem.

The biological motivation of introducing neuron division and budding into SN $\mathrm{P}$ systems comes from the recent discoveries in neurobiology related to neural stem cells - see, e.g., [4]. Neural stem cells persist throughout life within central nervous system in the adult mammalian brain, and this ensures a life-long contribution of new neurons to self-renewing nervous system with about 30000 new neurons being produced every day. Even in vitro, neural stem cells can be grown and extensively expanded for months. New neurons are produced by symmetric or asymmetric division. Two main neuron cell types are found: neuroblasts and astrocytes. The latter form a meshwork and are organized into channels. These observations are incorporated in SN P systems by considering neuron division and budding, and by providing a "synapse dictionary" according to which new synapses are generated, respectively.

The paper is organized as follows. In Section 2 we recall some mathematical preliminaries that will be used in the following. In Section 3 the formal definition of SN P systems with neuron division rules and neuron budding rules is given. In Section 4 we present a uniform family of SN P systems with neuron division and budding rules such that the systems can solve SAT problem in a polynomial time. 
Section 5 concludes the paper and suggests some possible open problems for future work.

\section{Prerequisites}

We assume the reader to be familiar with basic elements about membrane computing, e.g., from [13] and [14], and formal language theory, as available in many monographs. We mention here only a few notions and notations which are used through the paper.

For an alphabet $V, V^{*}$ denotes the set of all finite strings over $V$, with the empty string denoted by $\lambda$. The set of all nonempty strings over $V$ is denoted by $V^{+}$. When $V=\{a\}$ is a singleton, then we simply write $a^{*}$ and $a^{+}$instead of $\{a\}^{*}$, $\{a\}^{+}$.

A regular expression over an alphabet $V$ is defined as follows: (i) $\lambda$ and each $a \in V$ is a regular expression, (ii) if $E_{1}, E_{2}$ are regular expressions over $V$, then $\left(E_{1}\right)\left(E_{2}\right),\left(E_{1}\right) \cup\left(E_{2}\right)$, and $\left(E_{1}\right)^{+}$are regular expressions over $V$, and (iii) nothing else is a regular expression over $V$. With each regular expression $E$ we associate a language $L(E)$, defined in the following way: (i) $L(\lambda)=\{\lambda\}$ and $L(a)=\{a\}$, for all $a \in V$, (ii) $L\left(\left(E_{1}\right) \cup\left(E_{2}\right)\right)=L\left(E_{1}\right) \cup L\left(E_{2}\right), L\left(\left(E_{1}\right)\left(E_{2}\right)\right)=L\left(E_{1}\right) L\left(E_{2}\right)$, and $L\left(\left(E_{1}\right)^{+}\right)=\left(L\left(E_{1}\right)\right)^{+}$, for all regular expressions $E_{1}, E_{2}$ over $V$. Non-necessary parentheses can be omitted when writing a regular expression, and also $(E)^{+} \cup\{\lambda\}$ can be written as $E^{*}$.

\section{SN P Systems with Neuron Division and Budding}

As stated in the Introduction, SN P systems have been introduced in [6], in the framework of membrane computing. They can be considered as an evolution of $\mathrm{P}$ systems, corresponding to a shift from cell-like to neural-like architectures.

In SN P systems the cells (also called neurons) are placed in the nodes of a directed graph, called the synapse graph. The contents of each neuron consist of a number of copies of a single object type, called the spike. Every cell may also contain a number of firing and forgetting rules. Firing rules allow a neuron to send information to other neurons in the form of electrical impulses (also called spikes) which are accumulated at the target cell. The applicability of each rule is determined by checking the contents of the neuron against a regular set associated with the rule. In each time unit, if a neuron can use one of its rules, then one of such rules must be used. If two or more rules could be applied, then only one of them is non-deterministically chosen. Thus, the rules are used in the sequential manner in each neuron, but neurons function in parallel with each other. Note that, as usually happens in membrane computing, a global clock is assumed, marking the time for the whole system, and hence the functioning of the system is synchronized. When a cell sends out spikes it becomes "closed" (inactive) for a specified period 
of time, a fact which reflects the refractory period of biological neurons. During this period, the neuron does not accept new inputs and cannot "fire" (that is, emit spikes). Another important feature of biological neurons is that the length of the axon may cause a time delay before a spike arrives at the target. In SN P systems this delay is modeled by associating a delay parameter to each rule which occurs in the system. If no firing rule can be applied in a neuron, then there may be the possibility to apply a forgetting rule, that removes from the neuron a predefined number of spikes.

The structure of SN P systems (that is, the synapse graph) introduced in [6] is static. For both biological and mathematical motivations discussed in the Introduction, neuron division and budding are introduced into SN P systems. In this way, an exponential workspace can be generated in polynomial (even linear) time and computationally hard problems can be efficiently solved by means of a space-time tradeoff.

Formally, a spiking neural $P$ system with neuron division and budding of (initial) degree $m \geq 1$ is a construct of the form

$$
\Pi=\left(O, H, \text { syn }, n_{1}, \ldots, n_{m}, R, \text { in }, \text { out }\right),
$$

where:

1. $O=\{a\}$ is the singleton alphabet ( $a$ is called spike);

2. $H$ is a finite set of labels for neurons;

3. syn $\subseteq H \times H$ is a synapse dictionary, with $(i, i) \notin$ syn for $i \in H$;

4. $n_{i} \geq 0$ is the initial number of spikes contained in neuron $i, i \in\{1,2, \ldots, m\}$;

5. $R$ is a finite set of developmental rules, of the following forms:

(1) extended firing (also called spiking) rule $\left[E / a^{c} \rightarrow a^{p} ; d\right]_{i}$, where $i \in H, E$ is a regular expression over $a$, and $c \geq 1, p \geq 0, d \geq 0$, with the restriction $c \geq p$

(2) neuron division rule $[E]_{i} \rightarrow[]_{j} \|[]_{k}$, where $E$ is a regular expression and $i, j, k \in H$

(3) neuron budding rule $[E]_{i} \rightarrow[]_{i} /[]_{j}$, where $E$ is a regular expression and $i, j \in H$

6. in out $\in H$ indicate the input and the output neurons of $\Pi$.

Note that we have presented here an SN P system in a way slightly different from the usual definition present in the literature, where the neurons present initially in the system are explicitly listed as $\sigma_{i}=\left(n_{i}, R_{i}\right)$, where $1 \leq i \leq m$ and $R_{i}$ are the rules associated with neuron with label $i$. In what follows we will refer to neuron with label $i \in H$ also denoting it with $\sigma_{i}$.

It is worth to mention that by applying division rules different neurons can appear with the same label. In this context, $(i, j) \in$ syn means the following: there exist synapses from each neuron with label $i$ to each neuron with label $j$.

If an extended firing rule $\left[E / a^{c} \rightarrow a^{p} ; d\right]_{i}$ has $E=a^{c}$, then we will write it in the simplified form $\left[a^{c} \rightarrow a^{p} ; d\right]_{i}$; similarly, if a rule $\left[E / a^{c} \rightarrow a^{p} ; d\right]_{i}$ has $d=0$, then we can simply write it as $\left[E / a^{c} \rightarrow a^{p}\right]_{i}$; hence, if a rule $\left[E / a^{c} \rightarrow a^{p} ; d\right]_{i}$ has 
$E=a^{c}$ and $d=0$, then we can write $\left[a^{c} \rightarrow a^{p}\right]_{i}$. A rule $\left[E / a^{c} \rightarrow a^{p}\right]_{i}$ with $p=0$ is written in the form $\left[E / a^{c} \rightarrow \lambda\right]_{i}$ and is called extended forgetting rule. Rules of the types $\left[E / a^{c} \rightarrow a ; d\right]_{i}$ and $\left[a^{c} \stackrel{i}{\rightarrow} \lambda\right]_{i}$ are said to be standard.

If a neuron $\sigma_{i}$ contains $k$ spikes and $a^{k} \in L(E), k \geq c$, then the rule $\left[E / a^{c} \rightarrow\right.$ $\left.a^{p} ; d\right]_{i}$ is enabled and it can be applied. This means consuming (removing) $c$ spikes (thus only $k-c$ spikes remain in neuron $\sigma_{i}$ ); the neuron is fired, and it produces $p$ spikes after $d$ time units. If $d=0$, then the spikes are emitted immediately; if $d=1$, then the spikes are emitted in the next step, etc. If the rule is used in step $t$ and $d \geq 1$, then in steps $t, t+1, t+2, \ldots, t+d-1$ the neuron is closed (this corresponds to the refractory period from neurobiology), so that it cannot receive new spikes (if a neuron has a synapse to a closed neuron and tries to send a spike along it, then that particular spike is lost). In the step $t+d$, the neuron spikes and becomes open again, so that it can receive spikes (which can be used starting with the step $t+d+1$, when the neuron can again apply rules). Once emitted from neuron $\sigma_{i}$, the $p$ spikes reach immediately all neurons $\sigma_{j}$ such that there is a synapse going from $\sigma_{i}$ to $\sigma_{j}$ and which are open, that is, the $p$ spikes are replicated and each target neuron receives $p$ spikes; as stated above, spikes sent to a closed neuron are "lost", that is, they are removed from the system. In the case of the output neuron, $p$ spikes are also sent to the environment. Of course, if neuron $\sigma_{i}$ has no synapse leaving from it, then the produced spikes are lost. If the rule is a forgetting one of the form $\left[E / a^{c} \rightarrow \lambda\right]_{i}$, then, when it is applied, $c \geq 1$ spikes are removed. When a neuron is closed, none of its rules can be used until it becomes open again.

If (1) a neuron $\sigma_{i}$ contains $s$ spikes and $a^{s} \in L(E)$, and (2) there is no neuron $\sigma_{g}$ such that the synapse $(g, i)$ or $(i, g)$ exists in the system, for some $g \in\{j, k\}$, then the division rule $[E]_{i} \rightarrow[]_{j} \|[]_{k}$ is enabled and it can be applied. This means that consuming all these $s$ spikes the neuron $\sigma_{i}$ is divided into two neurons, $\sigma_{j}$ and $\sigma_{k}$. The new neurons contain no spike in the moment when they are created. They can have different labels, but they inherit the synapses that the father neuron already has (if there is a synapse from neuron $\sigma_{g}$ to the neuron $\sigma_{i}$, then in the process of division one synapse from neuron $\sigma_{g}$ to new neuron $\sigma_{j}$ and another one from $\sigma_{g}$ to $\sigma_{k}$ are established; similarly, if there is a synapse from the neuron $\sigma_{i}$ to neuron $\sigma_{h}$, then one synapse from $\sigma_{j}$ to $\sigma_{h}$ and another one from $\sigma_{k}$ to $\sigma_{h}$ are established). Note that the restriction provided by condition (2) to the use of the rule ensures that no synapse $(j, j)$ or $(k, k)$ appears. Except for the inheritance of synapses, the new neurons produced by division can have new synapses as provided by the synapse dictionary. Note that during the computation, it is possible that a synapse between neurons involved in the division rule and neurons existing in the system will appear that is not in the synapse dictionary syn, because of the inheritance of synapses. Therefore, the synapse dictionary syn has two functions: one is to deduce the initial topological structure of the SN P system (a directed graph), for example, if there are neurons $\sigma_{1}, \ldots, \sigma_{k}$ at the beginning of computation, then the initial topological structure of the system is $\operatorname{syn} \cap(\{1,2, \ldots, k\} \times\{1,2, \ldots, k\})$; another function is to guide the synapse establishment associated with the new 
neurons generated by neuron division or neuron budding. That is why we call syn a synapses dictionary.

If (1) a neuron $\sigma_{i}$ contains $s$ spikes, and $a^{s} \in L(E)$, and (2) there is no neuron $\sigma_{j}$ such that the synapse $(i, j)$ exists in the system, then the budding rule $[E]_{i} \rightarrow$ []$_{i} /[]_{j}$ is enabled and it can be applied. This means that consuming all the $s$ spikes a new neuron is created, $\sigma_{j}$. Both neurons are empty after applying the rule. The neuron $\sigma_{i}$ inherits the synapses going to it before using the rule. The neuron $\sigma_{j}$ created by budding by neuron $\sigma_{i}$ inherits the synapses going out of $\sigma_{i}$ before budding, that is, if there is a synapse from neuron $\sigma_{i}$ to some neuron $\sigma_{h}$, then a synapse from neuron $\sigma_{j}$ to neuron $\sigma_{h}$ is established (condition (2) ensures the fact that no synapse $(j, j)$ appears). There is also a synapse $(i, j)$ between neurons $\sigma_{i}$ and $\sigma_{j}$. Except for the above synapses associated with neurons $\sigma_{i}$ and $\sigma_{j}$, other synapses associated with neuron $\sigma_{j}$ can be established according to the synapses dictionary syn as in the case of neuron division rule.

In each time unit, if a neuron $\sigma_{i}$ can use one of its rules, then a rule from $R$ must be used. If several rules are enabled in neuron $\sigma_{i}$, irrespective of their types (spiking, dividing, or budding) then only one of them is chosen non-deterministically. When a spiking rule is used, the state of neuron $\sigma_{i}$ (open or closed) depends on the delay $d$. When a neuron division rule or neuron budding rule is applied, at this step the associated neuron is closed, it cannot receive spikes. In the next step, the neurons obtained by division or budding will be open and can receive spikes. Thus, the rules are used in the sequential manner in each neuron, but neurons function in parallel with each other.

It is worth noting here that the two neurons produced by a division rule can have labels different from each other and from the divided neuron, and that they are placed "in parallel", while in the budding case the old neuron (consumes all its spikes and) produces one new neuron which is placed "serially", after the neuron which budded.

The configuration of the system is described by the topology structure of the system, the number of spikes associated with each neuron, and the state of each neuron (open or closed). Using the rules as described above, one can define transitions among configurations. Any sequence of transitions starting in the initial configuration is called a computation. A computation halts if it reaches a configuration where all neurons are open and no rule can be used.

Traditionally, the input of an SN P system used in the accepting mode is provided in the form of a spike train, a sequence of steps when one spike or no spike enters the input neuron. In what follows we need several spikes at a time to come into the system via the input neuron, that is we consider "generalized spike trains", written in the form $a^{i_{1}} \cdot a^{i_{2}} \ldots \cdot a^{i_{r}}$, where $r \geq 1, i_{j} \geq 0$ for each $1 \leq j \leq r$. The meaning is that $i_{j}$ spikes are introduced in neuron $\sigma_{i n}$ in step $j$ (all these $i_{j}$ spikes are provided at the same time). Note that we can have $i_{j}=0$, which means that no spike is introduced in the input neuron. The period which separate the "packages" $a^{i_{j}}$ of spikes is necessary in order to make clear that we do not have 
here a concatenation of the strings describing these "packages", but a sequence of blocks (more formally, a sequence of multisets over the singleton alphabet $O$ ).

Spiking neural $\mathrm{P}$ systems can be used to solve decision problems, both in a semi-uniform and in a uniform way. When solving a problem $\mathcal{Q}$ in the semiuniform setting, for each specified instance $\mathcal{I}$ of $\mathcal{Q}$ an $\mathrm{SN} \mathrm{P}$ system $\Pi_{\mathcal{Q}, \mathcal{I}}$ is (i) built by a Turing machine in a polynomial time (with respect to the size of $\mathcal{I}$ ), (ii) its structure and initial configuration depend upon $\mathcal{I}$, and (iii) it halts (or emits a specified number of spikes in a given interval of time) if and only if $\mathcal{I}$ is a positive instance of $\mathcal{Q}$. On the other hand, a uniform solution of $\mathcal{Q}$ consists of a family $\left\{\Pi_{\mathcal{Q}}(n)\right\}_{n \in \mathbb{N}}$ of SN P systems that are built by a Turing machine in a polynomial time (with respect to the size $n$ ). When having an instance $\mathcal{I} \in \mathcal{Q}$ of size $n$, we introduce a polynomial (in $n$ ) number of spikes in a designated input neuron of $\Pi_{\mathcal{Q}}(n)$ and the computation halts (or, alternatively, a specified number of spikes is emitted in a given interval of time) if and only if $\mathcal{I}$ is a positive instance. The preference for uniform solutions over semi-uniform ones is given by the fact that they are more strictly related to the structure of the problem, rather than to specific instances. Indeed, in the semi-uniform setting we do not even need any input neuron, as the instance of the problem can be embedded into the initial configuration of the system from the very beginning.

\section{A Uniform Solution to SAT Problem}

Let us consider the NP-complete decision problem SAT [5]. The instances of SAT depend upon two parameters: the number $n$ of variables, and the number $m$ of clauses. We recall that a clause is a disjunction of literals, occurrences of $x_{i}$ or $\neg x_{i}$, built on a given set $X=\left\{x_{1}, x_{2}, \ldots, x_{n}\right\}$ of Boolean variables. Without loss of generality, we can avoid the clauses in which the same literal is repeated or both the literals $x_{i}$ and $\neg x_{i}$, for any $1 \leq i \leq n$, occur. In this way, a clause can be seen as a set of at most $n$ literals. An assignment of the variables $x_{1}, x_{2}, \ldots, x_{n}$ is a mapping $T: X \rightarrow\{0,1\}$ that associates to each variable a truth value. The number of all possible assignments to the variables of $X$ is $2^{n}$. We say that an assignment satisfies the clause $C$ if, assigned the truth values to all the variables which occur in $C$, the evaluation of $C$ (considered as a Boolean formula) gives 1 (true) as a result.

We can now formally state the SAT problem as follows.

Problem 1. NAME: SAT.

- INSTANCE: a set $C=\left\{C_{1}, C_{2}, \ldots, C_{m}\right\}$ of clauses, built on a finite set $\left\{x_{1}, x_{2}, \ldots, x_{n}\right\}$ of Boolean variables.

- QUESTION: is there an assignment of the variables $x_{1}, x_{2}, \ldots, x_{n}$ that satisfies all the clauses in $C$ ?

Equivalently, we can say that an instance of SAT is a propositional formula $\gamma_{n, m}=C_{1} \wedge C_{2} \wedge \cdots \wedge C_{m}$, expressed in the conjunctive normal form as a conjunction 
of $m$ clauses, where each clause is a disjunction of literals built using the Boolean variables $x_{1}, x_{2}, \ldots, x_{n}$. With a little abuse of notation, from now on we will denote by $\operatorname{SAT}(n, m)$ the set of instances of SAT which have $n$ variables and $m$ clauses.

Let us consider the polynomial time computable function $\langle n, m\rangle=((m+n)(m+$ $n+1) / 2)+m$ (the pair function), which is a primitive recursive and bijective function from $\mathbb{N}^{2}$ to $\mathbb{N}$. Let us build a uniform family $\left\{\Pi_{S A T}(\langle n, m\rangle)\right\}_{n, m \in \mathbb{N}}$ of SN P systems such that for all $n, m \in \mathbb{N}$ the system $\Pi_{S A T}(\langle n, m\rangle)$ solves all the instances of $S A T(n, m)$ in a number of steps which is linear in both $n$ and $m$. All the systems $\Pi_{S A T}(\langle n, m\rangle)$ will work in a deterministic way.

Because the construction is uniform, we need a way to encode any given instance $\gamma_{n, m}$ of $S A T(n, m)$. As stated above, each clause $C_{i}$ of $\gamma_{n, m}$ can be seen as a disjunction of at most $n$ literals, and thus for each $j \in\{1,2, \ldots, n\}$ either $x_{j}$ occurs in $C_{i}$, or $\neg x_{j}$ occurs, or none of them occurs. In order to distinguish these three situations we define the spike variables $\alpha_{i, j}$, for $1 \leq i \leq m$ and $1 \leq j \leq n$, as variables whose values are amounts of spikes; we assign to them the following values:

$$
\alpha_{i, j}=\left\{\begin{array}{l}
a, \text { if } x_{j} \text { occurs in } C_{i} ; \\
a^{2}, \text { if } \neg x_{j} \text { occurs in } C_{i} ; \\
a^{0}, \text { otherwise. }
\end{array}\right.
$$

In this way, clause $C_{i}$ will be represented by the sequence $\alpha_{i, 1} \cdot \alpha_{i, 2} \cdot \ldots \cdot \alpha_{i, n}$ of spike variables; in order to represent the entire formula $\gamma_{n, m}$ we just concatenate the representations of the single clauses, thus obtaining the generalized spike train $\alpha_{1,1} \cdot \alpha_{1,2} \cdot \ldots \cdot \alpha_{1, n} \cdot \alpha_{2,1} \cdot \alpha_{2,2} \cdot \ldots \cdot \alpha_{2, n} \cdot \ldots \cdot \alpha_{m, 1} \cdot \alpha_{m, 1} \cdot \ldots \cdot \alpha_{m, n}$. As an example, the representation of $\gamma_{3,2}=\left(x_{1} \vee \neg x_{2}\right)\left(x_{1} \vee x_{3}\right)$ is the sequence $a \cdot a^{2} \cdot a^{0} \cdot a \cdot a^{0} \cdot a$. In order to let the systems have enough time to generate necessary workspace before computing the instances of $S A T(n, m)$, a spiking train $\left(a^{0} \cdot\right)^{2 n}$ is added in front of the formula encoding spike train $\alpha_{1,1} \cdot \alpha_{1,2} \cdot \ldots \cdot \alpha_{1, n} \cdot \alpha_{2,1} \cdot \alpha_{2,2} \cdot \ldots \cdot \alpha_{2, n} \cdot \ldots$. $\alpha_{m, 1} \cdot \alpha_{m, 1} \cdot \ldots \cdot \alpha_{m, n}$. In general, for any given instance $\gamma_{n, m}$ of $S A T(n, m)$, the encoding sequence is $\operatorname{cod}\left(\gamma_{n, m}\right)=\left(a^{0} \cdot\right)^{2 n} \alpha_{1,1} \cdot \alpha_{1,2} \cdot \ldots \cdot \alpha_{1, n} \cdot \alpha_{2,1} \cdot \alpha_{2,2} \cdot \ldots \cdot \alpha_{2, n}$. $\ldots \cdot \alpha_{m, 1} \cdot \alpha_{m, 1} \cdot \ldots \cdot \alpha_{m, n}$.

For each $n, m \in \mathbb{N}$, we construct

$$
\Pi(\langle n, m\rangle)=\left(O, H, \text { syn }, n_{1}, \ldots, n_{q}, R, \text { in }, \text { out }\right),
$$

with the following components:

The initial degree of the system is $q=4 n+7$;

$$
\begin{aligned}
O= & \{a\} ; \\
H & =\{\text { in }, \text { out }, c l\} \cup\left\{d_{i} \mid i=0,1, \ldots, n\right\} \\
& \cup\left\{C x_{i} \mid i=1,2, \ldots, n\right\} \cup\left\{C x_{i} 0 \mid i=1,2, \ldots, n\right\} \\
& \cup\left\{C x_{i} 1 \mid i=1,2, \ldots, n\right\} \cup\left\{t_{i} \mid i=1,2, \ldots, n\right\} \\
& \cup\left\{f_{i} \mid i=1,2, \ldots, n\right\} \cup\{0,1,2,3\}
\end{aligned}
$$




$$
\begin{aligned}
\text { syn } & =\left\{\left(d_{i}, d_{i+1}\right) \mid i=0,1, \ldots, n-1\right\} \cup\left\{\left(d_{n}, d_{1}\right)\right\} \\
& \cup\left\{\left(i n, C x_{i}\right) \mid i=1,2, \ldots, n\right\} \cup\left\{\left(d_{i}, C x_{i}\right) \mid i=1,2, \ldots, n\right\} \\
& \cup\left\{\left(C x_{i}, C x_{i} 0\right) \mid i=1,2, \ldots, n\right\} \cup\left\{\left(C x_{i}, C x_{i} 1\right) \mid i=1,2, \ldots, n\right\} \\
& \cup\{(i+1, i) \mid i=0,1,2\} \cup\{(1,2),(0, \text { out })\} \\
& \cup\left\{\left(C x_{i} 1, t_{i}\right) \mid i=1,2, \ldots, n\right\} \cup\left\{\left(C x_{i} 0, f_{i}\right) \mid i=1,2, \ldots, n\right\} ;
\end{aligned}
$$

$n_{d_{0}}=n_{0}=n_{2}=n_{3}=1, n_{d_{1}}=6$, and there is no spike in the other neurons;

$R$ is the following set of rules:

(1) spiking rules:

$$
\begin{aligned}
& {[a \rightarrow a]_{i n},} \\
& {\left[a^{2} \rightarrow a^{2}\right]_{i n},} \\
& {[a \rightarrow a ; 2 n+n m]_{d_{0}},} \\
& {\left[a^{4} \rightarrow a^{4}\right]_{i}, i=d_{1}, \ldots, d_{n},} \\
& {\left[a^{5} \rightarrow \lambda\right]_{d_{1}}} \\
& {\left[a^{6} \rightarrow a^{4} ; 2 n+1\right]_{d_{1}},} \\
& {[a \rightarrow \lambda]_{C x_{i}}, i=1,2, \ldots, n,} \\
& {\left[a^{2} \rightarrow \lambda\right]_{C x_{i}}, i=1,2, \ldots, n,} \\
& {\left[a^{4} \rightarrow \lambda\right]_{C x_{i}}, i=1,2, \ldots, n,} \\
& {\left[a^{5} \rightarrow a^{5} ; n-i\right]_{C x_{i}}, i=1,2, \ldots, n,} \\
& {\left[a^{6} \rightarrow a^{6} ; n-i\right]_{C x_{i}}, i=1,2, \ldots, n,} \\
& {\left[a^{5} \rightarrow a^{4}\right]_{C x_{i} 1}, i=1,2, \ldots, n,} \\
& {\left[a^{6} \rightarrow \lambda\right]_{C x_{i} 1}, i=1,2, \ldots, n,} \\
& {\left[a^{5} \rightarrow \lambda\right]_{C x_{i} 0}, i=1,2, \ldots, n,} \\
& {\left[a^{6} \rightarrow a^{4}\right]_{C x_{i} 0}, i=1,2, \ldots, n,} \\
& {\left[\left(a^{4}\right)^{+} \rightarrow a\right]_{t_{i}}, i=1,2, \ldots, n,} \\
& {\left[\left(a^{4}\right)^{+} \rightarrow a\right]_{f_{i}}, i=1,2, \ldots, n,} \\
& {\left[a^{4 k-1} \rightarrow \lambda\right]_{t_{i}}, k=1,2, \ldots, n, i=1,2, \ldots, n,} \\
& {\left[a^{4 k-1} \rightarrow \lambda\right]_{f_{i}}, k=1,2, \ldots, n, i=1,2, \ldots, n,} \\
& {\left[a^{m} \rightarrow a^{2}\right]_{c l},} \\
& {\left[\left(a^{2}\right)^{+} / a \rightarrow a\right]_{\text {out }},} \\
& {[a \rightarrow a]_{i}, i=1,2,} \\
& {\left[a^{2} \rightarrow \lambda\right]_{2},} \\
& {[a \rightarrow a ; 2 n-1]_{3} ;}
\end{aligned}
$$

(2) neuron division rules:

$$
\begin{aligned}
& {[a]_{0} \rightarrow[]_{t_{1}} \|[]_{f_{1}},} \\
& {[a]_{t_{i}} \rightarrow[]_{t_{i+1}} \|[]_{f_{i+1}}, i=1,2, \ldots, n-1,} \\
& {[a]_{f_{i}} \rightarrow[]_{t_{i+1}} \|[]_{f_{i+1}}, i=1,2, \ldots, n-1}
\end{aligned}
$$

(3) neuron budding rules:

$[a]_{t_{n}} \rightarrow[]_{t_{n}} /[]_{c l}$,

$[a]_{f_{n}}^{t_{n}} \rightarrow[]_{f_{n}} /[]_{c l}$. 
The solution of the SAT problem is obtained by means of a brute force algorithm in the framework of SN P systems with neuron division and budding. Our strategy consists in the following phases:

- Generation Stage: The neuron division and budding are applied to generate an exponential number of neurons such that each possible assignment of variables $x_{1}, x_{2}, \ldots, x_{n}$ is represented by a neuron (with associated connections with other neurons by synapses).

- Input Stage: The system reads the encoding of the given instance of SAT.

- Satisfiability Checking Stage: The system checks whether or not there exists an assignment of variables $x_{1}, x_{2}, \ldots, x_{n}$ that satisfies all the clauses in the propositional formula $C$.

- Output Stage: According to the result of the previous stage, the system sends a spike to the environment if the answer is positive; otherwise, the system does not send any spike to the environment.

Let us have an overview of the computation. The initial structure of the system is shown in Figure 1 (in the figures which follow we only present the spiking and the forgetting rules, but not also the division and budding rules). The first three layers of the system constitutes the input module. The neuron $\sigma_{0}$ and its offsprings will be used to generate an exponential workspace by neuron division and budding rules. The auxiliary neurons $\sigma_{1}, \sigma_{2}$, and $\sigma_{3}$ supply necessary spikes to the neuron $\sigma_{0}$ and its offsprings for neuron division and budding rules. The neuron $\sigma_{\text {out }}$ is used to output the result.

Generation Stage: By the way of the encoding of instances, it is easy to see that the spike variables $\alpha_{i, j}$ will be introduced into neuron $\sigma_{i n}$ from step $2 n+1$ (it takes $2 n$ steps to read $\left(a^{0} \cdot\right)^{2 n}$ of $\left.\operatorname{cod}\left(\gamma_{n, m}\right)\right)$. In the first $2 n$ steps, the system generates an exponential workspace; after that, the system checks the satisfiability, and outputs the result.

The neuron $\sigma_{0}$ contains one spike, the rule $[a]_{0} \rightarrow[]_{t_{1}} \|[]_{f_{1}}$ is applied, and two neurons $\sigma_{t_{1}}$ and $\sigma_{f_{1}}$ are generated. They have the associated synapses $\left(1, f_{1}\right)$, $\left(1, t_{1}\right),\left(t_{1}\right.$, out $),\left(f_{1}\right.$, out $),\left(C x_{1} 1, t_{1}\right)$ and $\left(C x_{1} 0, f_{1}\right)$, where the first 4 synapses are obtained by the heritage of the synapses $(0$, out $)$ and $(1,0)$, respectively, and the last 2 synapses are established by the synapse dictionary. The auxiliary neuron $\sigma_{2}$ sends one spike to neuron $\sigma_{1}$, and at step 2 neuron $\sigma_{1}$ sends this spike to neurons $\sigma_{t_{1}}$ and $\sigma_{f_{1}}$ for the next division. At step 1 , the neuron $\sigma_{3}$ contains one spike, and the rule $[a \rightarrow a ; 2 n-1]_{3}$ is applied. It will send one spike to neuron $\sigma_{2}$ at step $2 n$ because of the delay $2 n-1$. (As we will see, at step $2 n$, neuron $\sigma_{1}$ also sends one spike to neuron $\sigma_{2}$, so neuron $\sigma_{2}$ will have 2 spikes, and the rule $\left[a^{2} \rightarrow \lambda\right]_{2}$ will be applied. In this way, after step $2 n$, the auxiliary neurons stop the work of supplying spikes for division and budding.) The structure of the system after step 1 is shown in Figure 2.

At step 2, neuron $\sigma_{1}$ sends one spike to neurons $\sigma_{2}, \sigma_{t_{1}}$, and $\sigma_{f_{1}}$. In the next step, neuron $\sigma_{2}$ sends one spike back to neuron $\sigma_{1}$; in this way, the auxiliary neurons $\sigma_{1}, \sigma_{2}$, and $\sigma_{3}$ supply spikes for division and budding every two steps in 


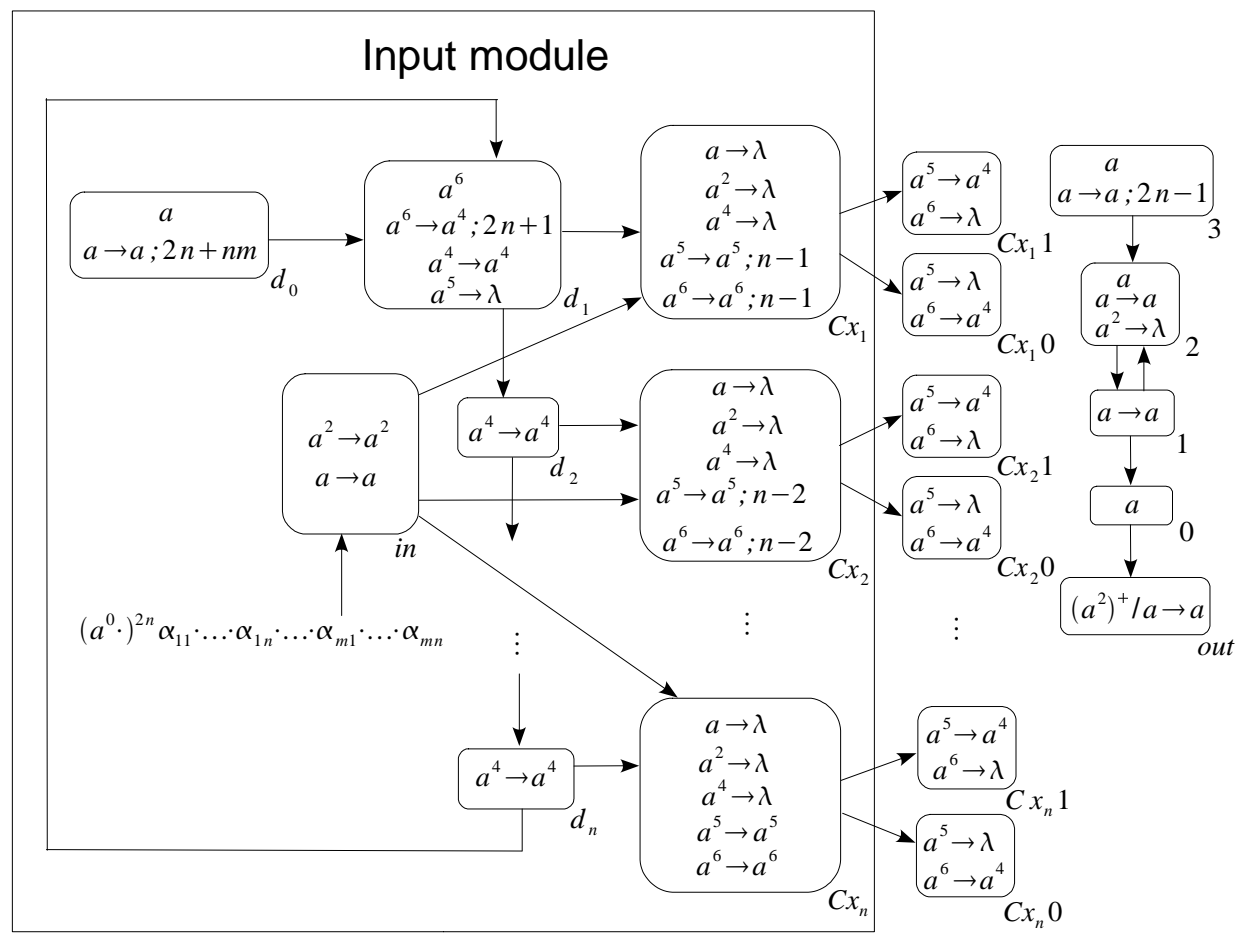

Fig. 1. The initial structure of system $\Pi(\langle n, m\rangle)$

the first $2 n$ steps. At step 3, only division rules can be applied in neurons $\sigma_{t_{1}}$ and $\sigma_{f_{1}}$, these two neurons are divided, and the associated synapses are obtained by heritage or synapse dictionary. The four neurons with labels $t_{2}$ or $f_{2}$ correspond to assignments where (1) $x_{1}=1$ and $x_{2}=1,(2) x_{1}=1$ and $x_{2}=0$, (3) $x_{1}=0$ and $x_{2}=1,(4) x_{1}=0$ and $x_{2}=0$, respectively. The neuron $C x_{1} 1$ (encoding that $x_{1}$ appears in a clause) has synapses from it to neurons whose corresponding assignments have $x_{1}=1$. That is, assignments with $x_{1}=1$ satisfy clauses where $x_{1}$ appears. The structure of the system after step 3 is shown in Figure 3. The neuron division is iterated until $2^{n}$ neurons with labels $t_{n}$ or $f_{n}$ appear at step $2 n-1$. The corresponding structure after step $2 n-1$ is shown in Figure 4.

At step $2 n$, each neuron with label $t_{n}$ or $f_{n}$ obtains one spike from neuron $\sigma_{1}$, then in next step the budding rules $[a]_{t_{n}} \rightarrow[]_{t_{n}} /[]_{c l}$ and $[a]_{f_{n}} \rightarrow[]_{f_{n}} /[]_{c l}$ are applied. Each created neuron $\sigma_{c l}$ has synapses $\left(t_{n}, c l\right)$ or $\left(f_{n}, c l\right)$ and $(c l$,out) by heritage. At step $2 n$, neuron $\sigma_{1}$ also sends one spike to neuron $\sigma_{2}$, at the time, neuron $\sigma_{3}$ sends one spike to neuron $\sigma_{2}$. So neuron $\sigma_{2}$ has two spikes, and the rule $\left[a^{2} \rightarrow \lambda\right]_{2}$ is applied at step $2 n+1$. After that, the auxiliary neurons cannot supply spikes any more, and the system passes to read the encoding of given instance. The structure of the system after step $2 n+1$ is shown in Figure 5 . 


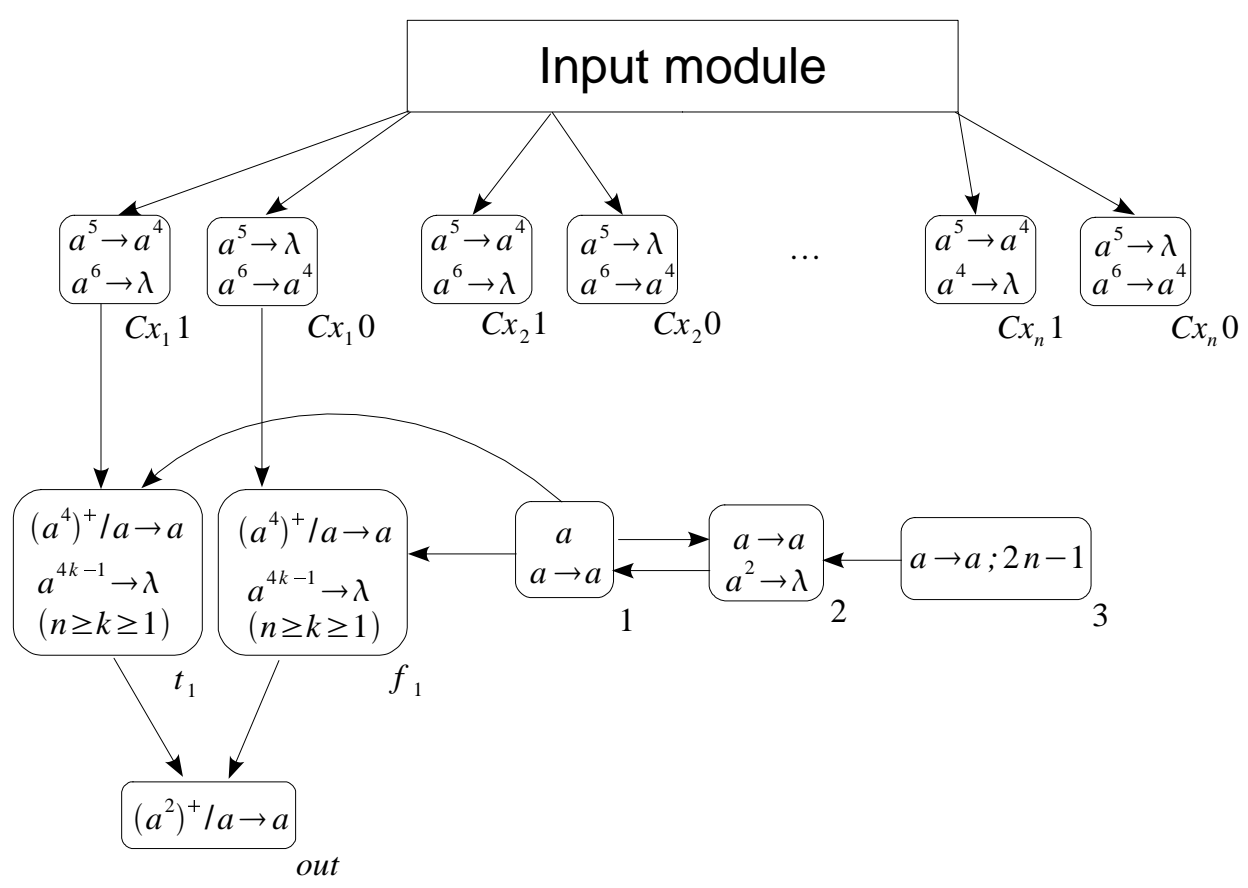

Fig. 2. The structure of system $\Pi(\langle n, m\rangle)$ after step 1

Input Stage: The input module consists of $2 n+2$ neurons, which are in the layers $1-3$ as illustrated in Figure $1 ; \sigma_{i n}$ is the unique input neuron. The spikes of the encoding sequence $\operatorname{code}\left(\gamma_{n, m}\right)$ are introduced into $\sigma_{i n}$ one "package" by one "package", starting from step 1 . It takes $2 n$ steps to introduce $\left(a^{0} \cdot\right)^{2 n}$ into neuron $\sigma_{i n}$. At step $2 n+1$, the value of the first spike variable $\alpha_{11}$, which is the virtual symbol that represents the occurrence of the first variable in the first clause, enters into neuron $\sigma_{i n}$. In the next step, the value of the spike variable $\alpha_{11}$ is replicated and sent to neurons $\sigma_{C x_{i}}$, for all $i \in\{1,2, \ldots, n\}$; in the meanwhile, neuron $\sigma_{d_{1}}$ send four auxiliary spikes to neurons $\sigma_{C x_{1}}$ and $\sigma_{d_{2}}$ (the rule $\left[a^{6} \rightarrow a^{4} ; 2 n+1\right]_{d_{1}}$ is applied at step 1). Hence, neuron $\sigma_{C x_{1}}$ will contain 4, 5 or 6 spikes: if $x_{1}$ occurs in $C_{1}$, then neuron $\sigma_{C x_{1}}$ collects 5 spikes; if $\neg x_{1}$ occurs in $C_{1}$, then it collects 6 spikes; if neither $x_{1}$ nor $\neg x_{1}$ occur in $C_{1}$, then it collects 4 spikes. Moreover, if neuron $\sigma_{C x_{1}}$ has received 5 or 6 spikes, then it will be closed for $n-1$ steps, according to the delay associated with the rules in it; on the other hand, if 4 spikes are received, then they are deleted and the neuron remains open. At step $2 n+3$, the value of the second spike variable $\alpha_{12}$ from neuron $\sigma_{i n}$ is distributed to neurons $\sigma_{C x_{i}}, 2 \leq i \leq n$, where the spikes corresponding to $\alpha_{11}$ are deleted by the rules $[a \rightarrow \lambda]_{C x_{i}}$ and $\left[a^{2} \rightarrow \lambda\right]_{C x_{i}}, 2 \leq i \leq n$. At the same time, the four auxiliary spikes are duplicated and one copy of them enters into neurons $\sigma_{C x_{2}}$ and $\sigma_{d_{3}}$, respectively. The neuron $\sigma_{C x_{2}}$ will be closed for $n-2$ steps only if it contains 


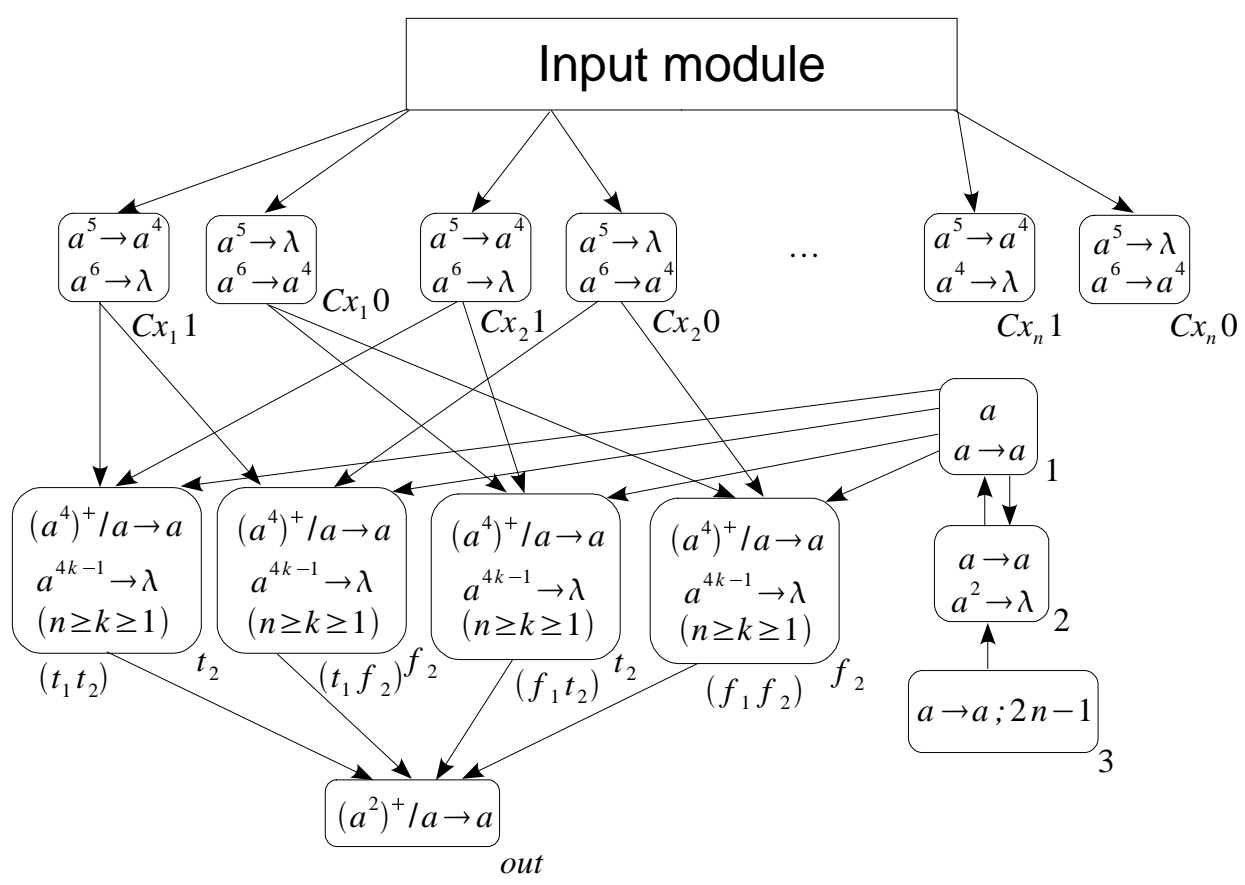

Fig. 3. The structure of system $\Pi(\langle n, m\rangle)$ after step 3

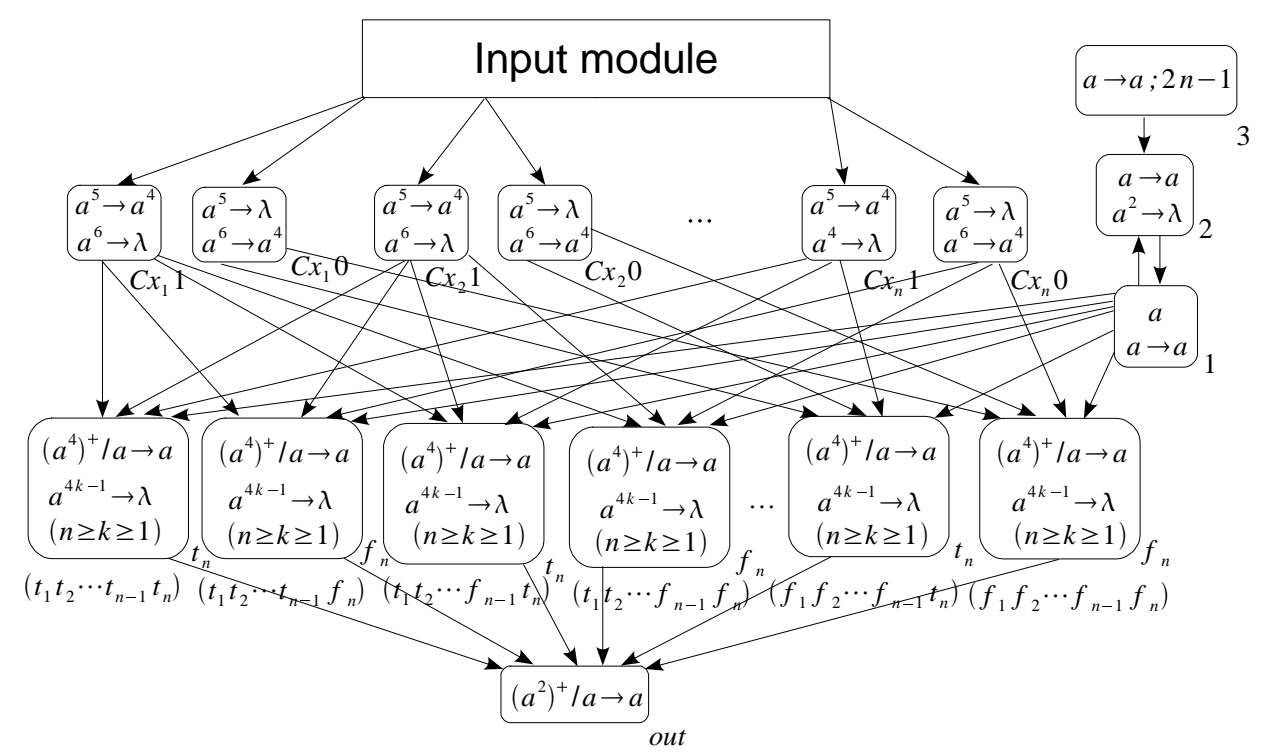

Fig. 4. The structure of system $\Pi(\langle n, m\rangle)$ after step $2 n-1$ 


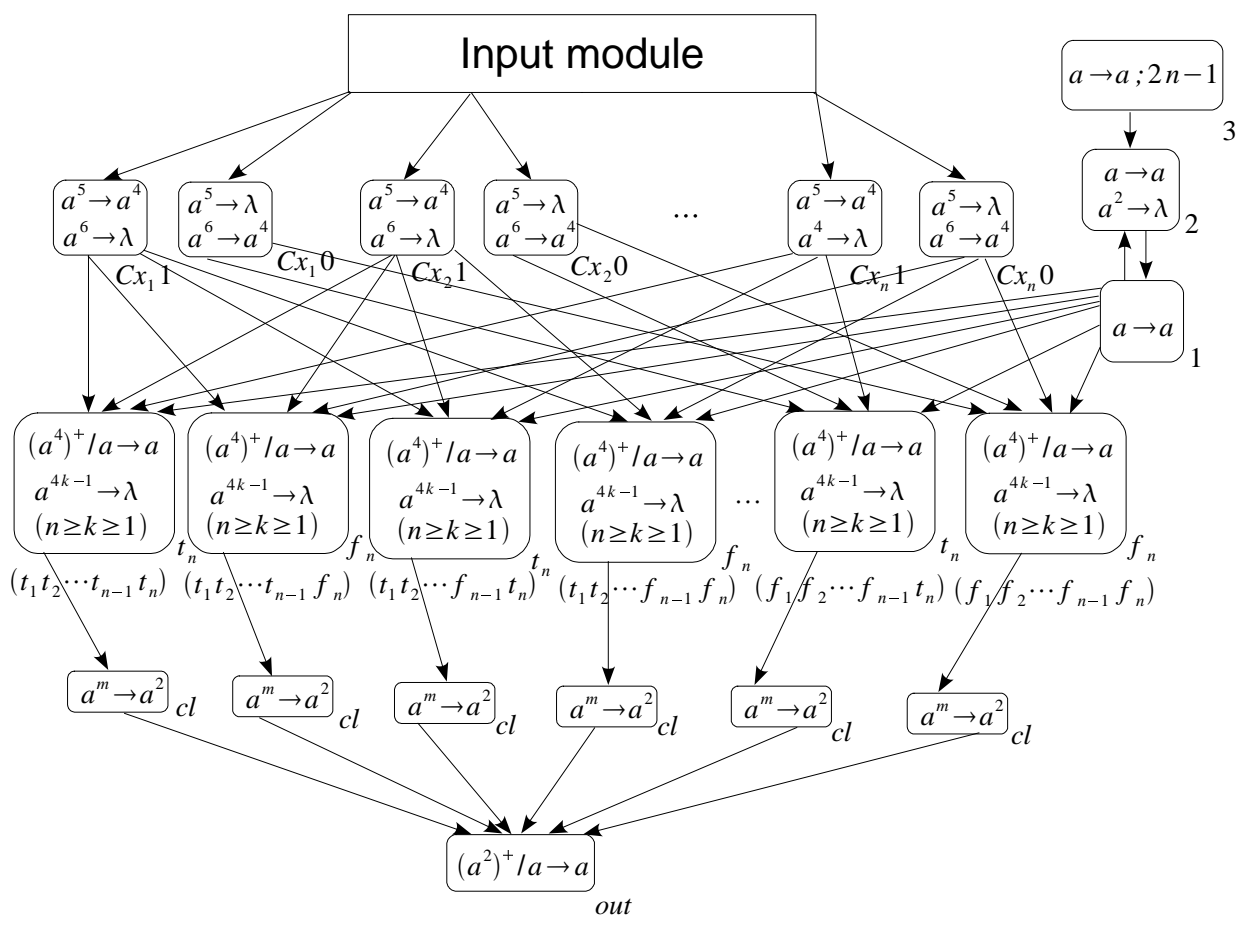

Fig. 5. The structure of system $\Pi(\langle n, m\rangle)$ after step $2 n+1$

5 or 6 spikes, which means that this neuron will not receive any spike during this period. In neurons $\sigma_{C x_{i}}, 3 \leq i \leq n$, the spikes represented by $\alpha_{12}$ are forgotten in the next step.

In this way, the values of the spike variables are introduced and delayed in the corresponding neurons until the value of the spike variable $\alpha_{1 n}$ of the first clause and the four auxiliary spikes enter together into neuron $\sigma_{C x_{n}}$ at step $3 n+1$. At that moment, the representation of the first clause of $\gamma_{n, m}$ has been entirely introduced in the system, and the second clause starts to enter into the input module. In general, it takes $m n+1$ steps to introduce the whole sequence $\operatorname{code}\left(\gamma_{n, m}\right)$ in the system, and the input process is completed at step $2 n+n m+1$.

At step $2 n+n m+1$, the neuron $\sigma_{d_{n}}$ sends four spike to neuron $\sigma_{d_{1}}$. At the same time, the auxiliary neuron $\sigma_{d_{0}}$ also sends a spike to the neuron $\sigma_{d_{1}}$ (the rule $[a \rightarrow a ; 2 n+n m]_{d_{0}}$ is used at the first step of the computation). So neuron $\sigma_{d_{1}}$ contains 5 spikes, and in the next step these 5 spikes are forgotten by the rule $\left[a^{5} \rightarrow \lambda\right]_{d_{1}}$. This ensures that the system eventually halts.

Satisfiability Checking Stage: Once all the values of spike variables $\alpha_{1 i}(1 \leq$ $i \leq n)$, representing the first clause, have appeared in their corresponding neurons $\sigma_{C x_{i}}$ in layer 3 , together with a copy of the four auxiliary spikes, all the spikes contained in $\sigma_{C x_{i}}$ are duplicated and sent simultaneously to the pair of neurons 
$\sigma_{C x_{i} 1}$ and $\sigma_{C x_{i} 0}$, for $i \in\{1,2, \ldots, n\}$, at step $3 n+2$. In this way, each neuron $\sigma_{C x_{i} 1}$ and $\sigma_{C x_{i} 0}$ receives 5 or 6 spikes when $x_{i}$ or $\neg x_{i}$ occurs in $C_{1}$, respectively, whereas it receives no spikes when neither $x_{i}$ nor $\neg x_{i}$ occurs in $C_{1}$. In general, if neuron $\sigma_{C x_{i} 1}$ receives 5 spikes, then the literal $x_{i}$ occurs in the current clause (say $C_{j}$ ), and thus the clause is satisfied by all those assignments in which $x_{i}$ is true. Neuron $\sigma_{C x_{i} 0}$ will also receive 5 spikes, but they will be deleted during the next computation step. On the other hand, if neuron $\sigma_{C x_{i} 1}$ receives 6 spikes, then the literal $\neg x_{i}$ occurs in $C_{j}$, and the clause is satisfied by those assignments in which $x_{i}$ is false. Since neuron $\sigma_{C x_{i} 1}$ is designed to process the case in which $x_{i}$ occurs in $C_{j}$, it will delete its 6 spikes. However, neuron $\sigma_{C x_{i} 0}$ will also have received 6 spikes, and this time it will send four spikes to those neurons which are bijectively associated with the assignments for which $x_{i}$ is false (refer to the generation stage for the corresponding synapses). In the next step, those neurons with label $t_{n}$ or $f_{n}$ that received at least four spikes send one spike to the corresponding neurons $\sigma_{c l}$ (the remaining spikes will be forgotten; note that the number of spikes received in neurons with label $t_{n}$ or $f_{n}$ is not more than $4 n$, because, without loss of generality, we assume that the same literal is not repeated and at most one of literals $x_{i}$ or $\neg x_{i}$, for any $1 \leq i \leq n$, can occur in a clause; that is, a clause is a disjunction of at most $n$ literals), with the meaning that the clause is satisfied by the assignments in which $x_{i}$ is false. This occurs in step $3 n+4$. Thus, the check for the satisfiability of the first clause has been performed; in a similar way, the check for the remaining clauses can proceed. All the clauses can thus be checked to see whether there exist assignments that satisfy all of them.

If there exist some assignments that satisfy all the clauses of $\gamma_{n, m}$, then the corresponding neurons with label $\mathrm{cl}$ succeed to accumulate $m$ spikes. Thus, the rule $\left[a^{m} \rightarrow a^{2}\right]_{c l}$ can be applied in these neurons. The satisfiability checking process is completed at step $2 n+m n+4$.

Output Stage: From the above explanation, it is not difficult to see that the output neuron receives spikes if and only if $\gamma_{n, m}$ is true. Furthermore, the output neuron sends exactly one spike to the environment at step $2 n+m n+6$ if and only if $\gamma_{n, m}$ is true.

From the previous explanations, one can see that the system correctly answers the question whether or not $\gamma_{n, m}$ is satisfiable. The duration of the computation is polynomial in term of $n$ and $m$ : if the answer is positive, then the system sends one spike to the environment at step $2 n+m n+6$; if the answer is negative, then the system halts in $2 n+m n+6$ steps, but does not send any spike to the environment.

Finally, we show that the family $\Pi=\{\Pi(\langle n, m\rangle) \mid n, m \in \mathbb{N}\}$ is polynomially uniform by deterministic Turing machines. We first note that the sets of rules associated with the system $\Pi(\langle n, m\rangle)$ are recursive. Hence, it is enough to note that the amount of necessary resources for defining each system is linear with respect to $n$, and this is indeed the case, since those resources are the following:

1. Size of the alphabet: $1 \in O(1)$.

2. Initial number of neurons: $4 n+7 \in O(n)$.

3. Initial number of spikes: $9 \in O(1)$. 
4. Number of labels for neurons: $6 n+8 \in O(n)$.

5. Size of synapse dictionary: $7 n+6 \in O(n)$.

6. Number of rules: $2 n^{2}+14 n+12 \in O\left(n^{2}\right)$.

\section{Conclusions and Remarks}

With computer science and biological motivation, neuron division and neuron budding are introduced into the framework of spiking neural $\mathrm{P}$ systems. We have proven that spiking neural $\mathrm{P}$ systems with neuron division and neuron budding can solve NP-complete problems in polynomial time. We exemplify this possibility with SAT problem.

Both neuron division rules and neuron budding rules can generate exponential workspace in linear time. In this sense, we used a double "power" to solve SAT problem in the systems constructed in this paper. It remains open to design efficient spiking neural P systems with either neuron division rules or neuron budding rules, but not both kinds of rules, for solving NP-complete problem.

Actually, we have here a larger set of problems. Besides the budding rules of the form considered above, we can also define "serial division rules", of the form $[E]_{i} \rightarrow[]_{j} /[]_{k}$, where $E$ is a regular expression and $i, j, k \in H$. The meaning is obvious: neuron $\sigma_{i}$ produces two neurons, $\sigma_{j}$ and $\sigma_{k}$, with possibly new labels $j, k$, linked by synapses as in the case of budding rules. Note that a budding rule is a particular case, with $i=j$. Thus, we can consider three types of rules: parallel division rules, budding rules (these two types were considered above), and serial division rules. Are rules of a single type sufficient in order to devise SN P systems which solve computationally hard problems in polynomial time?

For cell-like P systems, besides membrane division, there is another operation which was proved to provide ways to generate an exponential workspace in polynomial time, useful for trading space for time in solving NP-complete problem, namely membrane creation (a membrane is created from objects present in other membranes). Can this idea be also extended to SN P systems? Note that in the case of SN P systems we do not have neurons inside neurons, neither spikes outside neurons, hence this issue does not look easy to handle; maybe further ingredients should be added, such as glia cells, astrocytes, etc.

\section{Acknowledgements}

Valuable comments from Jun Wang are greatly appreciated. The work of L. Pan was supported by National Natural Science Foundation of China (Grant Nos. 60674106, 30870826, 60703047, and 60803113), Program for New Century Excellent Talents in University (NCET-05-0612), Ph.D. Programs Foundation of Ministry of Education of China (20060487014), Chenguang Program of Wuhan (200750731262), HUST-SRF (2007Z015A), and Natural Science Foundation of 
Hubei Province (2008CDB113 and 2008CDB180). The work of the last two authors was supported by Project of Excellence with Investigador de Reconocida Valia, from Junta de Andalucia, grant P08 - TIC 04200.

\section{References}

1. H. Chen, R. Freund, M. Ionescu, Gh. Păun, M.J. Pérez-Jiménez: On string languages generated by spiking neural P systems. Fundamenta Informaticae, 75 (2007), 141162.

2. H. Chen, M. Ionescu, T.-O. Ishdorj: On the efficiency of spiking neural P systems. Proceedings of the $8^{\text {th }}$ International Conference on Electronics, Information, and Communication, Ulanbator, Mongolia, June 2006, 49-52.

3. H. Chen, M. Ionescu, T.-O. Ishdorj, A. Păun, Gh. Păun, M.J. Pérez-Jiménez: Spiking neural $\mathrm{P}$ systems with extended rules: universality and languages. Natural Computing, 7, 2 (2008), 147-166.

4. R. Galli, A. Gritti, L. Bonfanti, A.L. Vescovi: Neural stem cells: an overview. Circulation Research, 92 (2003), 598-608.

5. M.R. Garey, D.S. Johnson: Computers and Intractability. A Guide to the Theory of NP-completeness. W.H. Freeman and Company, San Francisco, 1979.

6. M. Ionescu, Gh. Păun, T. Yokomori: Spiking neural P systems. Fundamenta Informaticae, 71, 2-3 (2006), 279-308.

7. T.-O. Ishdorj, A. Leporati: Uniform solutions to SAT and 3-SAT by spiking neural P systems with pre-computed resources. Natural Computing, 7, 4 (2008), 519-534.

8. A. Leporati, M.A. Gutiérrez-Naranjo: Solving Subset Sum by spiking neural P systems with pre-computed resources. Fundamenta Informaticae, 87, 1 (2008), 61-77.

9. A. Leporati, G. Mauri, C. Zandron, Gh. Păun, M.J. Pérez-Jiménez: Uniform solutions to SAT and Subset Sum by spiking neural P systems. Natural Computing, online version (DOI: 10.1007/s11047-008-9091-y).

10. A. Leporati, C. Zandron, C. Ferretti, G. Mauri: Solving numerical NP-complete problem with spiking neural P systems. Lecture Notes in Computer Sci., 4860 (2007), 336-352.

11. A. Leporati, C. Zandron, C. Ferretti, G. Mauri: On the computational power of spiking neural P systems. International Journal of Unconventional Computing, 2007, in press.

12. A. Păun, Gh. Păun: Small universal spiking neural P systems. BioSystems, 90, 1 (2007), 48-60.

13. Gh. Păun: Membrane Computing. An Introduction. Springer-Verlag, Berlin, 2002.

14. The P systems Website: http://ppage.psystems.eu. 
